International Journal of Pure and Applied Mathematics

Volume 93 No. 5 2014, 603-611

ISSN: 1311-8080 (printed version); ISSN: 1314-3395 (on-line version)

url: http://www.ijpam.eu

doi: http://dx.doi.org/10.12732/ijpam.v93i5.1

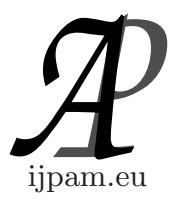

\title{
ON COMMUTATIVITY IN PRIME T-NEAR-RINGS WITH SYMMETRIC BI-DERIVATIONS
}

\author{
Kalyan Kumar Dey ${ }^{1}$, Akhil Chandra Paul ${ }^{2}$, Isamiddin S. Rakhimov ${ }^{3}$ \\ ${ }^{1,2}$ Department of Mathematics \\ Rajshahi University \\ Rajshahi, 6205, BANGLADESH \\ ${ }^{3}$ Department of Mathematics \\ FS \& Institute for Mathematical Research \\ (INSPEM) Universiti Putra Malaysia, MALAYSIA
}

\begin{abstract}
In this paper, we investigate the conditions of a prime $\Gamma$ - near-ring to be commutative by means of symmetric bi-derivations. We prove that under certain conditions, prime $\Gamma$-near-rings will be the commutative $\Gamma$ rings.
\end{abstract}

AMS Subject Classification: 16Y30, 16Y99

Key Words: $\Gamma$-near-ring, prime $\Gamma$-near-ring, symmetric bi-derivation

\section{Introduction}

The derivations in $\Gamma$-near-rings have been introduced by Bell and Mason [2]. They investigated some basic properties of derivations in $\Gamma$-near-rings. Then Mustafa [11] obtained some commutativity conditions for a $\Gamma$-near-ring with derivations. Some characterizations of $\Gamma$-near-rings and some regularity conditions were obtained by Cho [5]. M. Kazaz and Akin Alkan [12] introduced the notion of two-sided $\Gamma$ - $\alpha$-derivation of a $\Gamma$-near-ring and investigated the com-

Received: March 26, 2012

(C) 2014 Academic Publications, Ltd.

${ }^{\S}$ Correspondence author url: www.acadpubl.eu 
mutativity of a prime and semiprime $\Gamma$-near-rings. M Uckun, M. A. Ozturk and Y. B. Jun [13] worked on prime $\Gamma$-near-rings with derivations and they investigated the conditions for a $\Gamma$-near-ring to be commutative.

In this paper, the commutativity conditions are investigated for $\Gamma$-nearrings with symmetric bi-derivations. We prove that every prime $\Gamma$-near-ring is a commutative $\Gamma$-ring by means of the trace of symmetric bi-derivations.

\section{Preliminaries}

A $\Gamma$-near-ring is a triple $(N,+, G)$, where:

(i) $(N,+)$ is a group (not necessarily Abelian),

(ii) $G$ is a non-empty set of binary operations on $N$ such that for each $\alpha \in G,(N,+, \alpha)$ is a left near-ring.

(iii) $x \alpha(y \beta z)=(x \alpha y) \beta z, \forall x, y, z \in N$ and $\alpha, \beta \in G$.

Exactly speaking, it is a left $\Gamma$-near-ring because it satisfies the left distributive law. We will use the word $\Gamma$-near-ring to mean left G-near-ring. For a near-ring $N$, the set $N_{0}=\{x \in N: 0 \alpha x=0, \alpha \in G\}$ is called the zero-symmetric part of $N$. A $\Gamma$-near-ring $N$ is said to be zero-symmetric if $N=N_{0}$. $N$ will be prime, that is, will have the property that $x G N G y=0$ for $x, y \in N$ implies $x=0$ or $y=0$. The symbol $Z$ will denote the multiplicative center of $N$. For $x, y \in N$ and $\alpha \in G$, the symbol $[x, y]_{\alpha}$ will denote the commutator $x \alpha y-y \alpha x$, while the symbol $(\mathrm{x}, \mathrm{y})$ will denote the additivegroup commutator $x+y-x-y$. A mapping $D: N \times N \AA N$ is said to be symmetric if $D(x, y)=D(y, x), \forall x, y \in N$. A mapping $d: N 囚 N$ defined by $d(x)=D(x, x)$ is called the trace of $D$, where $D: N \times N \AA N$ is a symmetric mapping. It is obvious that, if $D: N \times N \mathrm{~B} N$ is a symmetric mapping which is also bi-additive (i.e., additive in both arguments), then the trace of $D$ satisfies the relation $d(x+y)=d(x)+2 D(x, y)+d(y), \forall x, y \in N$. A symmetric bi-additive mapping $D: N \times N 囚 N$ is called a symmetric bi-derivation if $D(x \alpha y, z)=D(x, z) \alpha y+x \alpha D(y, z)$ is fulfilled $\forall x, y, z \in N, \alpha \in G$. Then, for any $y \in N$, a mapping $x \AA D(x, y)$ is a derivation. Let $D$ be a symmetric bi-additive mapping of $N$. Then, $D(0, y)=0$ is fulfilled for all $y \in N$ and so $D(-x, y)=-D(x, y), \forall x, y \in N$. Therefore, the mapping $d: N 囚 N$ defined by $d(x)=D(x, x)$ is an even function. As usual, an element $c \in N$ for which $d(c)=D(c, c)=0$ is called a constant. 


\section{Main Results}

We need the following lemmas to obtain our main results

Lemma 3.1. Let $N$ be a prime $\Gamma$-near-ring.

(i) If $z \in Z-\{0\}$, then $z$ is not a zero divisor.

(ii) If $Z-\{0\}$ contains an element $z$ for which $z+z \in Z$, then $(N,+)$ is Abelian.

Proof. (i) If $z \in C-\{0\}$ and $z \alpha x=0, x \in N, \alpha \in G$, then $z \alpha r \beta x=0, x, r \in$ $N, \alpha \in G$. Thus we get $z G N G x=0$, by primeness of $N, x=0$.

(ii) Let $z \in C-\{0\}$ be an element such that $z+z \in C$, and let $x, y \in N, \alpha \in$ $G$. Since $z+z$ is distributive we get

$(x+y) \alpha(z+z)=x \alpha(z+z)+y \alpha(z+z)=x \alpha z+x \alpha z+y \alpha z+y \alpha z=z \alpha(x+x+y+y)$.

On the other hand

$$
(x+y) \alpha(z+z)=(x+y) \alpha z+(x+y) \alpha z=z \alpha((x+y+x+y) .
$$

Thus, $x+x+y+y=x+y+x+y$ and therefore $x+y=y+x$. Hence $(N,+)$ is Abelian.

Lemma 3.2. Let $N$ be a 2-torsion free G-near-ring, $D$ a symmetric biadditive mapping of $N$ and $d$ the trace of $D$. If $\mathrm{d}(\mathrm{x})=0$ for all $\mathrm{x} \in \mathrm{N}$, then $\mathrm{D}$ $=0$.

Proof. For any $x, y \in N, d(x+y)=d(x)+2 D(x, y)+d(y)$ and so, from the hypothesis and since $N$ is 2-torsion free, we get $D(x, y)=0 \forall x, y \in N$. Thus we get that $D=0$.

Lemma 3.3. Let $N$ be a 2 -torsion free prime $G$-near-ring, $D$ a symmetric bi-derivation of $N$ and $d$ the trace of $D$. If $x \alpha d(y)=0, \forall x, y \in N, \alpha \in G$, then $x=0$ or $D=0$.

Proof. For any $y, z \in N, d(y+z)=d(y)+2 D(y, z)+d(z)$. Now $x \alpha d(y+z)=$ $x \alpha d(y)+2 x \alpha D(y, z)+x \alpha d(z), \forall x, y, z \in N, \alpha \in G$, so, from the hypothesis and since $N$ is 2-torsion free, we get for all $x, y, z \in N, \alpha \in G$,

$$
x \alpha D(y, z)=0 .
$$

Taking $y \beta w, \beta \in G$, instead of $y$ in (1), $0=x \alpha D(y \beta w, z)=x \alpha D(y, z) \beta w+$ $x \alpha y \beta D(w, z)$ and so from (1) we get $x \alpha y \beta D(w, z)=0, \forall x, y, z, w \in N, \alpha, \beta \in G$. Since $N$ is a prime $\Gamma$-near-ring, we get that $x=0$ or $D=0$. 
Lemma 3.4. Let $N$ be a $\Gamma$-near-ring, $D$ a symmetric bi-additive mapping of $N$. Then the following are equivalent:

(i) $D(x \alpha y, z)=D(x, z) \alpha y+x \alpha D(y, z), \forall x, y, z \in N, \alpha \in G$.

(ii) $D(x \alpha y, z)=x \alpha D(y, z)+D(x, z) \alpha y, \forall x, y, z \in N, \alpha \in G$.

Proof. $(i i) \Rightarrow(i)$ Suppose $D(x \alpha y, z)=D(x, z) \alpha y+x \alpha D(y, z), \forall x, y, z \in$ $N, \alpha \in G$. Then

$$
\begin{aligned}
D(x \alpha(y+y), z)=D(x, z) \alpha(y+y)+x \alpha D(y+y, z) & \\
& =D(x, z) \alpha y+D(x, z) \alpha y+x \alpha D(y, z)+x \alpha D(y, z),
\end{aligned}
$$

and

$$
\begin{aligned}
D(x \alpha(y+y), z)=D(x \alpha y+x \alpha y, z) & =D(x \alpha y, z)+D(x \alpha y, z) \\
=D(x, z) \alpha y & +x \alpha D(y, z)+D(x, z) \alpha y+x \alpha D(y, z) .
\end{aligned}
$$

Therefore we get $D(x, z) \alpha y+x \alpha D(y, z)=x \alpha D(y, z)+D(x, z) \alpha y, \forall x, y, z \in$ $N, \alpha \in G$ and so we get that $D(x \alpha y, z)=x \alpha D(y, z)+D(x, z) \alpha y, \forall x, y, z \in$ $N, \alpha \in G$.

$(i) \Rightarrow(i i)$. This is proved in a similar way.

Lemma 3.5. Let $N$ be a $\Gamma$-near-ring, $D$ a symmetric bi-additive mapping of $N$ and $d$ the trace of $D$. Then we have, $\forall x, y, z, w \in N, \alpha, \beta \in G$ :

(i) $(D(x, z) \alpha y+x \alpha D(y, z)) \beta w=D(x, z) \alpha y \beta w+x \alpha D(y, z) \beta w$.

(ii) $(d(x) \alpha y+x \alpha D(x, y)) \beta w=d(x) \alpha y \beta w+x \alpha D(x, y) \beta w$.

(iii) $(x \alpha D(y, z)+D(x, z) \alpha y) \beta w=x \alpha D(y, z) \beta w+D(x, z) \alpha y \beta w$.

(iv) $(x \alpha D(x, z)+d(x) \alpha y) \beta w=x \alpha D(x, y) \beta w+d(x) \alpha y \beta w$.

Proof. (i) From the associative law, we have

$$
\begin{gathered}
D(x \alpha y, z)=D(x, z) \alpha y+x \alpha D(y, z), \forall x, y, z \in N, \alpha \in G, D((x \alpha y) \beta w, z) \\
=D(x \alpha y, z) \beta w+x \alpha y \beta D(w, z)=(D(x, z) \alpha y+x \alpha D(y, z)) \beta w+x \alpha y \beta D(w, z),
\end{gathered}
$$

and

$$
\begin{aligned}
& D(x \alpha(y \beta w), z)=D(x, z) \alpha y \beta w+x \alpha D(y \beta w, z) \\
& =D(x, z) \alpha y \beta w+x \alpha D(y, z) \beta w+x \alpha y \beta D(w, z) .
\end{aligned}
$$


Comparing the two expression we get, $\forall x, y, z, w \in N, \alpha, \beta \in G,(D(x, z) \alpha y+$ $x \alpha D(y, z)) \beta w=D(x, z) \alpha y \beta w+x \alpha D(y, z) \beta w$.

(ii) Taking $x$ instead of $z$ in (i) and since $D$ is a symmetric we get, $\forall x, y, w \in$ $N, \alpha, \beta \in G,(d(x) \alpha y+x \alpha D(x, y)) \beta w=d(x) \alpha y \beta w+x \alpha D(x, y) \beta w$.

The proof of (iii) (by Lemma 3.4) and (iv) are straightforward.

Theorem 3.1. Let $N$ be a prime $\Gamma$-near-ring, $D$ a non-zero symmetric bi-derivation of $N$ and $d$ the trace of $D$. If $N$ is 2-torsion free and $d(N) Z$, then $N$ is a commutative $\Gamma$-ring.

Proof. Let $c$ be an arbitrary constant and $x$ a non-constant. Then $d(x+c)=$ $d(x)+2 D(x, c)+d(c) \operatorname{in} Z$ and so, since $d(x) \in Z-\{0\}$ and $N$ is 2-torsion free, we get,

$$
D(x, c) \in Z-\{0\} .
$$

Taking $c \alpha x, \alpha \in G$, instead of $x$ in (2) we get, $D(c \alpha x, c)=c \alpha D(x, c)+$ $D(c, c) \alpha x=c \alpha D(x, c) \in Z-\{0\}$. From (2), it follows easily that $c \in Z-\{0\}$. Since $d(c+c)=0$, for all constant $c$, so from Lemma 2.1(ii), we get that $(N,+)$ is Abelian. In this case, suppose 0 is the only constant, since $d(x) \in Z, \forall x \in N$, we get for all $x, y \in N$,

$$
D(x, y) \in Z \text {. }
$$

Now, suppose that $u$ is not a zero divisor for $u \in N$ and let $x, y \in N, \alpha \in G$. Then

$$
\begin{aligned}
& D(u \alpha(x+u), y)=D(u, y) \alpha(x+u)+u \alpha D(x+u, y) \\
& \quad=D(u, y) \alpha x+D(u, y) \alpha u+u \alpha D(x, y)+u \alpha D(u, y)
\end{aligned}
$$

and

$$
\begin{aligned}
& D(u \alpha(x+u), y)=D(u \alpha x+u \alpha u, y)=D(u \alpha x, y)+D(u \alpha u, y) \\
& =D(u, y) \alpha x+u \alpha D(x, y)+D(u, y) \alpha u+u \alpha D(u, y) .
\end{aligned}
$$

Comparing the two expression we get, for all $x, y \in N, \alpha \in G$,

$$
D(u, y) \alpha u+u \alpha D(x, y)=u \alpha D(x, y)+D(u, y) \alpha u .
$$

From (3) and this equation we get, for all $x, y \in N, \alpha \in G$,

$$
0=u \alpha(D(u, y)+D(x, y)-D(x, y)-D(u, y))=u \alpha D(u+x-u-x, y) .
$$


Thus, since $u$ is not zero divisor, we get, for all $x, y \in N$.

$$
D((u, x), y)=0
$$

Taking $(u, x)$ instead of $y$ in (4) we get $d((u, x))=0, \forall x \in N$, and so $(u, x)$ is constant, i.e., $(u, x)=0, \forall x \in N$. Thus $u \in C(N)$ which is center of $(N,+)$. Now, let $x$ be a non-zero element of $N$. From the hypothesis and Lemma 3.3 $d(x)$ is not a zero divisor, we get $d(x) \in C(N), \forall 0 x \in N$. Since $d(x+z)=$ $d(x)+2 D(x, z)+d(z) \in C(N)$, where $0 z \in N$, we get $D(x, z) \in C(N), \forall 0 x$ and $0 y \in N$. Thus $0=D(x, z)+D(y, z)-D(x, z)-D(y, z)=D((x, y), z)$ and so, we get that $(x, y)=0, \forall x, y \in N$, i.e., $(N,+)$ is Abelian. Taking $x \beta w, \beta \in$ $G$, instead of $x$ in $(3)$, we have, $z \alpha(D(x, y) \beta w+x \alpha D(w, y))=(D(x, y) \beta w+$ $x \alpha \beta D(w, y)) z, \forall x, y, z, w \in N$. Thus from (3), Lemma 3.4 and Lemma 3.5 we get, $\forall x, y, z, w \in N, \alpha, \beta \in G$,

$$
D(x, y) \beta[z, w] \alpha=D(w, y) \beta[z, x] \alpha .
$$

Taking $d(w)$ instead of $w$ in (5). From the hypothesis we get, for all $x, y, z, w \in$ $N, \alpha, \beta \in G$,

$$
D(d(w), y) \beta[z, x] \alpha=0 .
$$

Substituting $y d r$ for $y$, where $r \in N$, in (6) and from (6) we get, for all $x, y, z, w \in N, \alpha, \beta, d \in G$,

$$
D(d(w), y) d r \beta[z, x] \alpha=0 .
$$

Now, suppose that $N$ is not commutative. In this case, since $N$ is prime and from (6) we get, for all $y, w \in N$

$$
D(d(w), y)=0
$$

Substituting $x+w$ for $w$ in (8), since $N$ is a 2-torsion free and from (8) we get, for all $x, y, w \in N$

$$
D(D(x, w), y)=0 .
$$

Substituting $x l w$ for $x$ in (9) and so, from (8) and (9) we get, for all $x, y, w \in$ $N, l \in G$,

$$
D(x, w) l D(w, y)+D(x, y) l d(w)=0
$$

Substituting $x$ for $w$ in (10) and since $N$ is 2 -torsion free, we get, for all $x, y \in$ $N, l \in G$,

$$
D(x, y) l d(x)=0
$$


Taking $x g y$ instead of $y$ in (11), from (11) we get, for all $x, y \in N, l, g \in G$,

$$
d(x) \operatorname{lyg} d(x)=0 .
$$

From (12) and since $N$ is 3-prime we get that $d(x)=0$. By Lemma 3.2 we have $D=0$. But this is a contradiction.

Theorem 3.2. Let $N$ be a prime $\Gamma$-near-ring, $D$ a non-zero symmetric biderivation of $N$ and $d$ the trace of $D$. If $N$ is 2-torsion free and $d(y), d(y)+d(y) \in$ $C(D(x, y))$, forallx $y, z \in N$, then $N$ is a commutative G-ring.

Proof. From the hypothesis, if both $w$ and $w+w$ commute element-wise with $D(x, z), \forall x, y \in N, \alpha \in G$, then $(D(x, z)+D(y, z)) \alpha(w+w)=(D(x, z)+$ $D(x, z)+D(y, z)+D(y, z)) \alpha w$.

On the other hand

$$
(D(x, z)+D(y, z)) \alpha(w+w)=(D(x, z)+D(y, z)+D(x, z)+D(y, z)) \alpha w .
$$

Comparing the two expression we get, for all $x, y \in N, \alpha \in G, D(x+y-x-$ $y) \alpha w=0$. This implies that

$$
D((x, y), z) \alpha w=0 .
$$

Thus, let $w=d(r)$ in (13), we get $D((x, y), z) \alpha d(r)=0, \forall x, y, z, r \in N$ and so, $D((x, y), z)=0, \forall x, y, z, \in N$ by Lemma 3.3. Since $z \alpha(x, y)$ is also an additive commutator for any $z \in N, \alpha \in G$, we have $0=D(z \alpha(x, y), z)=$ $d(z) \alpha(x, y), \alpha \in G$, and so $(x, y)=0$ by Lemma 3.3. That is, $(N,+)$ is Abelian. Now, since $d(y) \in C(D(x, y)) \forall x, y, z \in N, \alpha \in G$, we have

$$
[D(x, z), d(y)] \alpha=0 .
$$

Thus, replacing $z$ by $z \beta w$ in (14), and from (14), we get, for all $x, y, z, w \in$ $N, \alpha, \beta \in G$,

$$
\begin{aligned}
& 0=D(x, z \beta w) \alpha d(y)-d(y) \alpha D(x, z \beta w) \\
& \quad=(D(x, z) \beta w+z \beta D(x, w)) \alpha d(y)-d(y) \alpha(D(x, z) \beta w+z \beta D(x, w)),
\end{aligned}
$$

and so, from (14) and Lemma 3.5(i), since $(N,+)$ is Abelian, we get for all $x, y, z, w \in N, \alpha, \beta \in G$,

$0=D(x, z) \beta w \alpha d(y)-D(x, z) \alpha d(y) \beta w+z \beta d(y) \alpha D(x, w)-d(y) \alpha z \beta D(x, w)$.

Taking $d(z)$ for $z$ in the previous equation, and the hypothesis, we get,

$$
\forall x, y, z, w \in N, \alpha, \beta \in G, D(x, d(z)) \beta[w, d(y)] \alpha=0 .
$$


Replacing $x$ by $x d r$ in (15), and from (15), we get, $\forall x, y, z, w \in N, \alpha, \beta, d \in G$,

$$
D(x, d(z)) d r \beta[w, d(y)] \alpha=0 .
$$

From (16) and since $N$ is prime $\Gamma$-near-ring we get that $[w, d(y)] \alpha=0, \forall y, w \in$ $N, \alpha \in G$, or $D(x, d(z))=0, \forall x, z \in N$. If $d(N) Z$, then $N$ is commutative $\Gamma$-ring by Theorem 3.6. If $D(d(z), x)=0, \forall x, z \in N$, then the argument used in the proof of Theorem 3.6 (see, equation (8)) shows that $D=0$. But this is a contradiction.

\section{References}

[1] H. E. Bell and M. N. Daif, On derivations and commutativity in prime rings, Acta. Math. Hungar. 66 (1995), no. 4, 337-343.

[2] H. E. Bell and G. Mason, On derivations in near-rings. In: Gerhard Betsch (Ed.), Near-Rings and Near-Fields, Proceedings of the conference held at the University of Tubingen, Tubingen, August 4-10, 1985 (pp. 31\{35). Noth-Holland, Amsterdam, 1987.

[3] H. E. Bell and G. Mason, On derivations in near-rings and rings. Math. J. Okayama Univ. 34 (1992), 135-144.

[4] J. Bergen: Derivations in prime rings. Canad. Math. Bull. 26 (1983), no. $3,267-227$.

[5] Y. U. Cho: A study on derivations in near-rings. Pusan Kyongnam Math. J. 12 (1996), no. 1, 63-69.

[6] I. N. Herstein: A note on derivations. Canad. Math. Bull. 21 (1978), no. 3, 369-370.

[7] J. D. P. Meldrum: Near-Rings and Their Links with Groups, Research Notes in Mathematics, 134. Pitman (Advanced Publishing Program), Boston-London-Melbourne, 1985. MR 88a:16068.

[8] G. Pilz: Near-rings, North-Holland Mathematics Studies, 23. NorthHolland, Amsterdam, 1983.

[9] E. C. Posner: Derivations in prime rings. Proc. Amer. Math. Soc. 8 (1957), 1093-1100. 
[10] Yong Uk Cho, Some conditions on derivations in prime near rings, J Korea Soc Math Educ Ser B Pure Appl Math 8, 2 2001, 145-152.

[11] Mustafa Asci, $\Gamma$-(s,t)-Derivation on $\Gamma$ Near Ring, International Math Forum, 2, 3, 2007, 97-102.

[12] Mustafa Kazaz and Akin Alkan, Two sided $\Gamma$ - $\alpha$-derivations in prime and semiprime $\Gamma$-near-rings, Commun Korean Math. Soc. 23, 4, 2008, 469-477.

[13] Y. Bae Jun, K. Ho Kim and Y. Uk Cho, On $\Gamma$-derivation in $\Gamma$-near-rings, Soochow Journal of Mathematics, 29, 3, 2003, 275-282. 
